\title{
WEIGHTED WEAK-TYPE $(1,1)$ INEQUALITIES FOR ROUGH OPERATORS
}

\author{
STEVE HOFMANN
}

(Communicated by J. Marshall Ash)

Abstract. Let $\Omega$ be homogeneous of degree 0 , have mean value 0 on the circle, and belong to $L^{q}\left(S^{1}\right), 1<q \leq \infty$. Then the two-dimensional operator defined by

$$
T f(x)=\text { "pv" } \int \Omega(y)|y|^{-2} f(x-y) d y
$$

is shown to be of weak-type $(1,1)$ with respect to the weighted measures $|x|^{\alpha} d x$, if $-2+1 / q<\alpha<0$. Under the weaker assumption that $\Omega$ belongs to $L \log ^{+} L\left(S^{1}\right)$, the same result holds if $-1<\alpha<0$. Similar results are also obtained for the related maximal operator

$$
M_{\Omega} f(x)=\sup _{r>0} r^{-2} \int_{|y|<r}|\Omega(y) f(x-y)| d y .
$$

\section{INTRODUCTION AND STATEMENT OF RESULTS}

In 1957, E. M. Stein [S2] showed that if $T f(x)=\operatorname{pv} \int K(x, y) f(y) d y$ is bounded on $L^{p}\left(R^{n}, d x\right), 1<p<\infty$, and if $|K(x, y)| \leq A|x-y|^{-n}$, then $T$ is also bounded on $L^{p}\left(|x|^{\alpha} d x\right),-n<\alpha<n(p-1)$. In particular, Stein's result holds for $K(x, y)=\Omega((x-y) /|x-y|)|x-y|^{-n}$, with $\Omega$ in $L^{\infty}(|x|=1)$. In 1971 , under the weaker hypothesis that $\Omega \in L \log ^{+} L(|x|=1)$, Muckenhoupt and Wheeden [MW] showed that $T$ is still bounded on $L^{p}\left(|x|^{\alpha} d x\right)$ for the restricted range $-1<\alpha<p-1$, a result that could also be obtained by the method of rotations. The advantage of the technique used in [MW] is that it also yielded $L^{p}$ boundedness of $T$ for $\Omega$ in $L^{q}, 1<q<\infty$, for an intermediate range of $\alpha$ (depending on $q$ ) between those obtained for $\Omega$ in $L \log ^{+} L$ and $\Omega$ in $L^{\infty}$. Several years later, Kurtz and Wheeden [KW] proved an analogous result for the case $p=1$, namely that $T$ is of weak-type $(1,1)$ with respect to the measure $|x|^{\alpha} d x,-1<\alpha<0$, but with the additional assumption that $\Omega$ satisfies the "integral Dini" condition

$$
\infty>\int_{0}^{1} \omega(\delta) \delta^{-1} d \delta, \quad \text { where } \omega(\delta)=\sup \int_{|x|=1}\left|\Omega\left(\rho x^{\prime}\right)-\Omega\left(x^{\prime}\right)\right| d x^{\prime},
$$

Received by the editors October 3, 1988, and in revised form February 28, 1989. 1980 Mathematics Subject Classification (1985 Revision). Primary 42B20. 
the sup running over all rotations $\rho$ of magnitude $|\rho| \leq \delta$. (Here, of course, the size condition on $\Omega$ can be reduced to integrability, since $L^{1}+$ integral Dini implies $L \log ^{+} L$ [CWZ]). The main result of this paper is that for $n=2$, the $L^{1}$ theory can be brought in line with the $L^{p}$ theory, (i.e. we obtain weak $(1,1)$ bounds with respect to weights $|x|^{\alpha}$ without integral Dini).

For a nonnegative weight function $w$, define $\|f\|_{p, w}=\left(\int|f|^{p} w d x\right)^{1 / p}$, and for a Lebesgue measurable set $E$ let $w(E)=\int_{E} w d x$. In two dimensions we have the following:

Theorem 1. Let $\Omega$ be homogeneous of degree 0 , and have mean value 0 . Let $K(y)=\Omega(y)|y|^{-2}$, and define $T_{\varepsilon} f(x)=\int_{|y|>\varepsilon} K(y) f(x-y) d y$. Set $w(x)=$ $|x|^{\alpha}$. Then for

$$
\Omega \in L \log ^{+} L\left(S^{1}\right) \text { and }-1<\alpha<0,
$$

and

$$
\Omega \in L^{q}\left(S^{1}\right), 1<q \leq \infty, \text { and }-2+1 / q<\alpha<0,
$$

there exists $c$ independent of $\varepsilon$ such that

$$
w\left\{x \in \mathbf{R}^{2}:\left|T_{\varepsilon} f(x)\right|>\lambda\right\} \leq c \lambda^{-1}\|f\|_{1, w},
$$

for all $\lambda>0$.

Remarks.

(i) That $(*)$ holds when $\alpha=0$ is already known (see $[\mathrm{H}]$ for $\Omega \in L^{q}$, and [CRdF] for $\Omega \in L \log ^{+} L$ ).

(ii) Theorem 1(a) is almost sharp (i.e. except for $\alpha=-1$ ), in the sense that for $\alpha<-1$ or $\alpha>0$, there exist $\Omega \in L \log ^{+} L$ and integral Dini for which (*) fails (see $[\mathrm{KW}])$.

(iii) The key estimate needed to prove Theorem 1 is $(0.4)$ of $\S 0$. It is enough to prove (0.4) for the case $q=\infty$. Part (a) then follows by the same argument used in [CRdF], and part (b), $1<q<\infty$, follows by an interpolation in the spirit of that in Theorem 2 of [C].

The proof of Theorem 1 will also contain the proof of the following:

Theorem 2. Let $w$ and $\Omega$ be as in Theorem 1 (except that $\Omega$ need not have mean value zero). Set $M_{\Omega} f(x)=\sup _{r>0} r^{-2} \int_{|y|<r}|\Omega(y) f(x-y)| d y$. Then (*) holds for $M_{\Omega}$ in place of $T_{\varepsilon}$.

Remarks.

(iv) For bounded $\Omega, M_{\Omega}$ is dominated by the Hardy-Littlewood maximal function, so in this case it is already well known that $M_{\Omega}$ is weak $(1,1)$ with respect to any $A_{1}$ weight $w$. 
(v) Theorem 2 is also true for $\alpha=0$ in $\mathbf{R}^{\mathbf{n}}$ (see [C] when $n=2$, [CRdF] for the general case).

(vi) Theorem 2 is actually a consequence of the "estimate for the diagonal terms" (see Lemma 1, which is stated in the next section), by the same reasoning as in [C, Theorem 2]. The details are left to the reader. Thus only Theorem 1 need be proved explicitly.

\section{Proof of Theorem 1: The basic setup}

Let $f$ belong to $L^{1}(w d x)$, and fix $\lambda>0$. We begin with a dyadic Whitney decomposition [S1, chapter 6] of the set $\{\mathrm{Mf}>\lambda\}$ (where $M f$ is the HardyLittlewood maximal function), so that $\{\mathrm{Mf}>\lambda\}$ is equal to the union of nonoverlapping dyadic cubes $\left\{Q_{n}\right\}$. As in [S1, chapter 1], we can write $f=$ $g+b$, where $\|g\|_{2, w} \leq c\left(\lambda\|f\|_{1, w}\right)^{1 / 2}$ and $b=\sum b_{n}$, with $b_{n}$ supported in $Q_{n},\left\|b_{n}\right\|_{1} \leq c \lambda\left|Q_{n}\right|$, and $\int b_{n}=0$. By the $L^{p}$ results of [MW], it is enough to prove

$$
w\left\{x \in \mathbf{R}^{2}:\left|T_{\varepsilon} b(x)\right|>\lambda\right\} \leq c \lambda^{-1}\|b\|_{1, w} .
$$

For a cube $Q$, let $k Q$ have the same center as $Q$, but side length $k$ times as large, and let $E$ be the union of $\left\{40 Q_{n}\right\}$. As is well known, it is enough to show that

$$
w\left\{x \in E^{c}:\left|T_{\varepsilon} b(x)\right|>\lambda\right\} \leq c \lambda^{-1}\|b\|_{1, w} .
$$

Choose a nonnegative $C_{0}^{\infty}(\mathbf{R})$ function $\phi$, supported in the interval $\left(\frac{1}{4}, 1\right)$, such that $\sum_{j \in \mathbf{Z}} \phi\left(2^{-j} x\right)=1$ for all $x>0$. Define $K_{j}(x)=\Omega(x)|x|^{-2}$. $\phi\left(2^{-j}|x|\right)$, and let $B_{i}=\sum b_{n}$, the sum running over those $n$ for which $Q_{n}$ has side length $2^{i}$. Then

$$
\left|T_{\varepsilon} b\right| \leq\left|\sum_{i} \sum_{j: 2^{j-2}>\varepsilon} B_{i} * K_{j}\right|+\sum_{i} \sum_{j: 2^{j} \approx \varepsilon}\left|B_{i}\right| *\left|K_{j}\right| .
$$

There are at most three $j$ terms appearing in the second sum, which will therefore be bounded in $L^{1}(w d x)$ norm by $c\|\Omega\|_{q}\|b\|_{1, w}$, (if $\alpha>-2+1 / q$, $1 \leq q \leq \infty)$ once we show that

$$
\left\|\left|B_{i}\right| *\left|K_{j}\right|\right\|_{1, w} \leq c\|\Omega\|_{q}\left\|B_{i}\right\|_{1, w}, \quad \text { for } \alpha>-2+1 / q, 1 \leq q \leq \infty .
$$

The estimate $(0.2)$ can be obtained by straightforward arguments from the following special case of Lemma 1 from [MW]:

Lemma 0. Let $\Omega$ be homogeneous of degree 0 . For $\alpha>-2+1 / q, 1 \leq q \leq \infty$,

$$
\int_{|y| \leq a|x|}|\Omega(y)||y+x|^{\alpha} d y \leq c\|\Omega\|_{q}|x|^{\alpha+2} .
$$

Lemma 0 follows from a change to polar coordinates and the inequality

$$
\begin{aligned}
\int_{0}^{a|x|}\left|s y^{\prime}+x\right|^{\alpha} d s & \leq c|x|^{\alpha+1}\left(1+\left|x^{\prime}+y^{\prime}\right|^{\alpha+1}\right), \quad \alpha \neq-1 \\
& \leq c \log ^{+}\left(1 /\left|x^{\prime}+y^{\prime}\right|\right), \quad \alpha=-1,
\end{aligned}
$$


where $x^{\prime}=x /|x|$ and $y^{\prime}=y /|y|$. This is a special case of inequality (2.4) from [MW], which is proved on page 252 of that paper.

To handle the rest of $T_{\varepsilon} b$ we make the change of index $s=j-i$, and write

$$
\sum_{i} \sum_{j: 2^{j-2}>\varepsilon} B_{i} * K_{j}(x)=\sum_{s} \sum_{j: 2^{j-2}>\varepsilon} B_{j-s} * K_{j}(x),
$$

which for $x$ in $E^{\mathcal{c}}$ is equal to

$$
\sum_{s \geq 5} \sum_{j: 2^{j-2}>\varepsilon} B_{j-s} * K_{j}(x) .
$$

To see this, note that for $s<5$, and $b_{n}$ supported in $Q_{n}$ with side length $2^{j-s}$, we have $\left(b_{n} * K_{j}\right)$ supported in $40 Q_{n}$. Our aim will now be to show that for $\Omega \in L^{\infty}, \alpha>-2$, and for some $\eta>0$,

$$
\left\|\sum_{j: 2^{j-2}>\varepsilon} B_{j-s} * K_{j}\right\|_{2, w} \leq c\|\Omega\|_{\infty} 2^{-\eta s}\left(\lambda s\|b\|_{1, w}\right)^{\frac{1}{2}}
$$

The case $q=\infty$ of Theorem 1(b), follows immediately from(0.4), Minkowski's inequality, and Tchebychev's inequality. Furthermore, as mentioned in Remark (iii) of the Introduction, the rest of Theorem 1 follows from $(0.4)$ by previously developed techniques.

We now proceed to the proof of (0.4). Set $w(x)=|x|^{\alpha},-2<\alpha<0$, and write

$$
\begin{gathered}
\left\|\sum_{j} B_{j-s} * K_{j}\right\|_{2, w}^{2}=\sum_{j}\left\|B_{j-s} * K_{j}\right\|_{2, w}^{2} \\
+2 \sum_{j} \sum_{i=j-2}^{j-1}\left\langle B_{i-s} * K_{i}, B_{j-s} * K_{j}\right\rangle_{w}+2 \sum_{j} \sum_{i \leq j-3}\left\langle B_{j-s} * K_{j}, B_{i-s} * K_{i}\right\rangle_{w} \\
=I+I I+I I I,
\end{gathered}
$$

where $\langle u, v\rangle_{w}=\int u v w d x$. The required estimate for $I$ (and hence for $I I$ by Schwarz's inequality) follows immediately from:

Lemma 1. There exists $\eta>0$ such that, for all fixed $s \geq 5$,

$$
\left\|B_{j-s} * K_{j}\right\|_{2, w}^{2} \leq c 2^{-\eta s} \lambda\|\Omega\|_{\infty}^{2}\left\|B_{j-s}\right\|_{1, w} .
$$

Lemma 1 will be proved in $\S 1$.

We now briefly discuss the cross terms. Set $K_{i}^{*}(x)=K_{i}(-x)$ and define $w_{x}(y)=w(x+y)$. Then $I I I$ is equal to twice

$$
\begin{gathered}
\sum \sum\left\langle B_{j-s}, K_{j}^{*} *\left[\left(B_{i-s} * K_{i}\right) w\right]\right\rangle \\
=\sum_{j} \sum_{i \leq j-3} \int B_{j-s}(x) \int K_{j}^{*}(x-y) \int K_{i}(y-z) B_{i-s}(z) d z w(y) d y d x \\
=\sum_{j} \int B_{j-s}(x) \int \sum_{i \leq j-3} B_{i-s}(z)\left(\left(K_{j} w_{x}\right) * K_{i}^{*}(z-x)\right) d z d x .
\end{gathered}
$$


Therefore the cross terms $I I I$ will also satisfy $(0.4)$ once we prove

Lemma 2. There exists $\eta>0$ such that, for all fixed $s \geq 5$, and each $x \in$ $\sup B_{j-s}$,

$$
\left|\int \sum_{i \leq j-3} B_{i-s}(z)\left(\left(K_{j} w_{x}\right) * K_{i}^{*}(z-x)\right) d z\right| \leq c \lambda 2^{-\eta s} s\|\Omega\|_{\infty}^{2} w(x) .
$$

The proof of Lemma 2 is in $\S 2$.

We now set some notation that will hold for the rest of this paper. For fixed $s \geq 5$, write $B_{j-s}=B_{1, j-s}+B_{2, j-s}$, where we define $B_{1, j-s}$ to be the sum of those $b_{n}$ whose supporting cubes are contained in the complement of $\left\{2^{j-3} \leq\right.$ $\left.|x| \leq 2^{j+1}\right\}$. Thus, every cube in $\sup B_{2, j-s}$ meets $\left\{2^{j-3} \leq|x| \leq 2^{j+1}\right\}$, and since $s>4, \sup B_{2, j-s}$ is contained in $\left\{2^{j-4} \leq|x| \leq 2^{j+2}\right\}$. It is enough to prove inequality $(0.4)$ for $B_{1, j-s}$ and $B_{2, j-s}$ separately.

\section{Proof of Lemma 1}

The square of the $L^{2}(w d x)$ norm of $B_{j-s} * K_{j}$ is equal to

$$
\int B_{j-s}(x) \int B_{j-s}(z)\left(K_{j} w_{x}\right) * K_{j}^{*}(z-x) d z d x .
$$

Recall that $w(x)=|x|^{\alpha},-2<\alpha<0$, and $w_{x}(y)=w(x+y)$. We may assume (and we will throughout this section) that $\|\Omega\|_{\infty}=1$. Also in this section, with $x$ and $j$ fixed, we set $H=\left(K_{j} w_{x}\right) * K_{j}^{*}$. To prove Lemma 1 , it is then enough to show:

$$
\text { for each fixed } x \in \sup B_{1, j-s}, \quad\left|\int B_{1, j-s}(z) H(z-x) d z\right| \leq c 2^{-\eta s} \lambda w(x) \text {, }
$$

for each fixed $x \in \sup B_{2, j-2}, \quad\left|\int B_{2, j-s}(z) H(z-x) d z\right| \leq c 2^{-\eta s} \lambda w(x)$.

We discuss (1.1) first. By definition of $\sup K_{j}$ and $\sup B_{1, j-s}$, for $x \in$ $\sup B_{1, j-s}$,

$$
|H(z-x)|=\left|\int K_{j}(y)\right| y+\left.\left.x\right|^{\alpha} K_{j}^{*}(z-x-y) d y\left|\leq 2^{-2 j}\right| x\right|^{\alpha} .
$$

The proof of (1.1) also uses the following weighted version of Lemma 2.2 of [C]:

Lemma 1.4. For fixed $x$ in $\sup B_{1, j-s}$ and for $\left|z-z_{0}\right| \leq|z-x| / 2$,

$$
\left|\left(K_{j} w_{x}\right) * K_{j}^{*}(z-x)-\left(K_{j} w_{x}\right) * K_{j}^{*}\left(z_{0}-x\right)\right| \leq c 2^{-j}|z-x|^{-4 / 3}\left|z-z_{0}\right|^{1 / 3} w(x) \text {. }
$$


The estimate (1.1) then follows from (1.3) and Lemma 1.4 in essentially the same way that in [C] Christ's Lemma 2.1 follows from his Lemma 2.2. The details are left to the reader. Before proving Lemma 1.4, we turn out attention to (1.2).

Set $y^{\prime}=y /|y|$. For fixed $x \in \sup B_{2, j-s}$, write $K_{j}(y)=K_{1, j, x}(y)+K_{2, j, x}(y)$, where $K_{1, j, x}(y)=\Omega\left(y^{\prime}\right)|y|^{-2} \phi\left(2^{-j}|y|\right) \chi\left(y^{\prime}\right)$. Here $\chi$ is the characteristic function of $\left\{\left\|y^{\prime}-x^{\prime}\right\|<2^{-\beta s}\right\}, \beta>0$ to be chosen, and $\left\|y^{\prime}-x^{\prime}\right\|$ is defined to be the minimum of $\left(\left|y^{\prime}-x^{\prime}\right|,\left|y^{\prime}+x^{\prime}\right|\right)$. For fixed $x$ and $j$, this splitting induces a corresponding decomposition $H=H_{1}+H_{2}$, with $H_{1}=\left(K_{1, j, x} w_{x}\right) * K_{j}^{*}$. Then $\left|H_{1}(z-x)\right|$ is bounded by

$$
\begin{aligned}
\int\left|K_{1, j, x}(y)\left\|K_{j}^{*}((z-x)-y)\right\| y+x\right|^{\alpha} d y & \\
& \leq 2^{-4 j} \int_{|y|<a|x|}\left|\chi\left(y^{\prime}\right)\right||y+x|^{\alpha} d y .
\end{aligned}
$$

Here we have used the fact that $K_{j}$ and $B_{2, j-s}$ are supported in annuli with inner and outer radii equal to a constant times $2^{j}$. By a change to polar coordinates, the last integral is bounded by

$$
2^{-4 j} a|x| \int_{|y|=1} \chi\left(y^{\prime}\right) \int_{0}^{a|x|}\left|s y^{\prime}+x\right|^{\alpha} d s d y^{\prime} .
$$

By the estimate $(0.3)$ of $\S 0$ applied to the inner integral, since $\alpha>-2$ and $|x| \approx 2^{j}$, we have

$$
\left|H_{1}(z-x)\right| \leq c 2^{-2 j}|x|^{\alpha} 2^{-\gamma \beta s},
$$

for some positive constant $\gamma$. Obtaining (1.2) with $H_{1}$ in place of $H$ is now easy. In fact, the required estimate follows in this case from (1.5) once we observe that $\left\|B_{2, j-s}\right\|_{1} \leq c \lambda \sum\left|Q_{n}\right|$, where all the $Q_{n}$ appearing in the sum are contained in a ball of radius $2^{j+2}$.

We now consider (1.2) for $H_{2}=\left(K_{2, j, x} w_{x}\right) * K_{j}^{*}$, which follows from

Lemma 1.6. For fixed $s$ and $j$, for fixed $x$ in $\sup B_{2, j-s}$, and for $\left|z-z_{0}\right| \leq$ $|z-x| / 2$,

$$
\left|H_{2}(z-x)-H_{2}\left(z_{0}-x\right)\right| \leq c 2^{-j+(1-\alpha) \beta s}|z-x|^{-4 / 3}\left|z-z_{0}\right|^{1 / 3}|x|^{\alpha},
$$

where $\beta$ is the same as in the definition of $K_{1, j, x}$ and $K_{2, j, x}$.

In fact, for appropriately chosen $\beta$, Lemma 1.6 implies (1.2) in the present case in exactly the same way that Lemma 1.4 implied (1.1), because in analogy with (1.3), $\left|H_{2}(z-x)\right|$ is bounded by

$$
2^{-2 j} \int\left|K_{2, j . x}(y) \| y+x\right|^{\alpha} d y \leq c 2^{-2 j} 2^{-\alpha \beta s}|x|^{\alpha} .
$$


The last inequality follows from the fact that

$$
|y+x| \geq c 2^{j}\left|y^{\prime}-x^{\prime}\right| \geq c 2^{j-\beta s} \geq c|x| 2^{-\beta s}
$$

by definition of the supports of $B_{2, j-s}$ and $K_{2, j, x}$.

The proof of Lemma 1 is now reduced to proving Lemmas 1.4 and 1.6.

Proof of Lemma 1.4. Set $\psi(t)=t^{-1} \phi(t)$. Then

$$
K_{j}(x)=\Omega(x)|x|^{-1} 2^{-j} \psi\left(2^{-j}|x|\right) .
$$

In analogy with Lemma 2.2 of [C], we have

$$
\left(K_{j} w_{x}\right) * K_{j}^{*}=\int_{S^{1}} \int_{S^{1}} \mu_{\theta, j, x} * \mu_{\omega, j}^{*} d \theta d \omega,
$$

where

$$
\begin{aligned}
\left\langle\mu_{\theta, j, x}, h\right\rangle & =\Omega(\theta) \int_{0}^{\infty} h(t \theta) w_{x}(t \theta) 2^{-j} \psi\left(2^{-j} t\right) d t \\
\left\langle\mu_{\omega, j}^{*}, h\right\rangle & =\Omega(\omega) \int_{0}^{\infty} h(-s \omega) 2^{-j} \psi\left(2^{-j} s\right) d s,
\end{aligned}
$$

and

$$
\begin{aligned}
\left\langle\mu_{\theta, j, x} *\right. & \left.\mu_{\omega, j}^{*}, h\right\rangle \\
& =2^{-2 j} \Omega(\theta) \Omega(\omega) \int_{0}^{\infty} \int_{0}^{\infty} h(t \theta-s \omega) w_{x}(t \theta) \psi\left(2^{-j} t\right) \psi\left(2^{-j} s\right) d t d s .
\end{aligned}
$$

By making the change of variable $z=t \theta-s \omega$, this last expression equals

$$
2^{-2 j} \Omega(\theta) \Omega(\omega) \Delta^{-1} \int h(z) w_{x}\left(\theta\left[\omega^{\perp} \cdot z\right] / \Delta\right) \psi\left(2^{-j}\left[\omega^{\perp} \cdot z\right] / \Delta\right) \psi\left(2^{-j}\left[\theta^{\perp} \cdot z\right] / \Delta\right) d z,
$$

where $\omega^{\perp}=\left(-\omega_{2}, \omega_{1}\right), \theta^{\perp}=\left(-\theta_{2}, \theta_{1}\right)$, and $\Delta=\left|\omega^{\perp} \cdot \theta\right|$. Thus

$$
\begin{aligned}
\mu_{\theta, j, x} * \mu_{\omega, j}^{*}(z-x)= & 2^{-2 j} \Omega(\theta) \Omega(\omega) \Delta^{-1} w_{x}\left(\theta\left[\omega^{\perp} \cdot(z-x)\right] / \Delta\right) \\
& \cdot \psi\left(2^{-j}\left[\omega^{\perp} \cdot(z-x)\right] / \Delta\right) \psi\left(2^{-j}\left[\theta^{\perp} \cdot(z-x)\right] / \Delta\right),
\end{aligned}
$$

and

$$
\left(K_{j} w_{x}\right) * K_{j}^{*}(z-x)=\int_{S^{1}} \int_{S^{1}} \mu_{\theta, j, x} * \mu_{\omega, j}^{*}(z-x) d \theta d \omega .
$$

It was Michael Christ who in Lemma 2.2 of [C] first observed that representations of this type could be used to obtain some smoothness from a convolution of kernels that are smooth only in the radial direction. In fact (with the normalization $\|\Omega\|_{\infty}=1$ ),

$$
\begin{aligned}
& \left|\nabla_{z} \mu_{\theta, j, x} * \mu_{\omega, j}^{*}(z-x)\right| \\
& \leq c \Delta^{-2}\left(2^{-3 j}\left|\left(\omega^{\perp} \cdot(z-x) / \Delta\right) \theta+x\right|^{\alpha}+2^{-2 j}\left|\left(\omega^{\perp} \cdot(z-x) / \Delta\right) \theta+x\right|^{\alpha-1}\right) \\
& \leq c \Delta^{-2} 2^{-3 j}|x|^{\alpha} .
\end{aligned}
$$


This last inequality follows from the fact that since $2^{j-2} \leq\left|\omega^{\perp} \cdot(z-x) / \Delta\right| \leq 2^{j}$ and $x \in \sup B_{1, j-s}$, we have $\left|\left(\omega^{\perp} \cdot(z-x) / \Delta\right) \theta+x\right|>c \max \left(|x|, 2^{j}\right)$. Thus

$$
\left|\left(\omega^{\perp} \cdot(z-x) / \Delta\right) \theta+x\right| 2^{-j}>c
$$

and

$$
\left|\left(\omega^{\perp} \cdot(z-x) / \Delta\right) \theta+x\right|^{\alpha} \leq c|x|^{\alpha} .
$$

By similar reasoning,

$$
\left|\mu_{\theta, j, x} * \mu_{\omega, j}^{*}(z-x)\right| \leq 2^{-2 j}|x|^{\alpha} \Delta^{-1} .
$$

For fixed $x$ in $\sup B_{1, j-s}$, following Lemma 2.2 of [C], we set

$$
H(y-x)=I_{\delta}(y)+J_{\delta}(y),
$$

where

$$
I_{\delta}(y)=\iint_{\|\theta-\omega\| \leq \delta} \mu_{\theta, j, x} * \mu_{\omega, j}^{*}(y-x) d \theta d \omega .
$$

Note that by geometry,

$$
\Delta \approx\|\theta-\omega\| .
$$

Then by (1.9), and by the support of $\mu_{\theta, j, x} * \mu_{\omega, j}^{*}$ (see (1.7)),

$$
\left|I_{\delta}(z)\right| \leq c|z-x|^{-2}|x|^{\alpha} \delta .
$$

Also, by (1.8)

$$
\left\|\nabla J_{\delta}\right\|_{\infty} \leq c 2^{-3 j}|x|^{\alpha} \delta^{-2}
$$

Now,

$$
\left|H(z-x)-H\left(z_{0}-x\right)\right| \leq\left|I_{\delta}(z)\right|+\left|I_{\delta}\left(z_{0}\right)\right|+\left|z-z_{0}\right|\left\|\nabla J_{\delta}\right\|_{\infty} .
$$

Since $\left|z-z_{0}\right| \leq|z-x| / 2$, we have $\left|I_{\delta}(z)\right| \approx\left|I_{\delta}\left(z_{0}\right)\right|$. Lemma 1.4 then follows, if we set $\delta=|z-x|^{2 / 3}\left|z-z_{0}\right|^{1 / 3} 2^{-j}$.

Proof of Lemma 1.6. As in the proof of Lemma 1.4, we have the representation

$$
\left(K_{2, j, x} w_{x}\right) * K_{j}^{*}(z-x)=\iint_{\left\|\theta-x^{\prime}\right\|>2^{-\beta s}} \mu_{\theta, j, x} * \mu_{\omega, j}^{*}(z-x) d \theta d \omega .
$$

The restriction on the domain of integration follows from the definition of $K_{2, j, x}$. For $x \in \sup B_{2, j-s}$, we have $|x| \approx 2^{j} \approx\left|\omega^{\perp} \cdot(z-x) / \Delta\right|$, so for $\left\|\theta-x^{\prime}\right\|>2^{-\beta s}$,

$$
\left|\left(\omega^{\perp} \cdot(z-x) / \Delta\right) \theta+x\right| \geq c 2^{j}\left\|\theta-x^{\prime}\right\| \geq c 2^{j-\beta s} \approx|x| 2^{-\beta s} .
$$

Thus $\left|\nabla_{z} \mu_{\theta, j, x} * \mu_{\omega, j}^{*}(z-x)\right|$ is bounded by

$$
\begin{gathered}
c \Delta^{-2}\left(2^{-3 j}\left|\left(\omega^{\perp} \cdot(z-x) / \Delta\right) \theta+x\right|^{\alpha}+2^{-2 j}\left|\left(\omega^{\perp} \cdot(z-x) / \Delta\right) \theta+x\right|^{\alpha-1}\right) \\
\leq c \Delta^{-2} 2^{-3 j+(1-\alpha) \beta s}|x|^{\alpha} .
\end{gathered}
$$

Similarly,

$$
\left|\mu_{\theta, j, x} * \mu_{\omega, j}^{*}(z-x)\right| \leq 2^{-2 j}|x|^{\alpha} \Delta^{-1} 2^{-\alpha \beta s} .
$$


We set $\left(K_{2, j, x} w_{x}\right) * K_{j}^{*}(y-x)=I_{\delta, \beta}(y)+J_{\delta, \beta}(y)$, where

$$
I_{\delta, \beta}(y)=\iint_{\|\theta-\omega\| \leq \delta} \mu_{\theta, j, x} * \mu_{\omega, j}^{*}(y-x) \chi(\theta) d \theta d \omega .
$$

Here $\chi(\theta)=1$ if $\left\|\theta-x^{\prime}\right\|>2^{-\beta s}$, and 0 otherwise. Lemma 1.6 then follows in exactly the same way as Lemma 1.4, and with the same $\delta$. This concludes the proof of Lemma 1.

\section{Proof of Lemma 2}

To prove Lemma 2, it is enough to prove the following (as above $\|\Omega\|_{\infty}=1$ ): (2.1) for each fixed $x \in \sup B_{1, j-s}$,

$$
\left|\int \sum_{i \leq j-3} B_{1, i-s}(z)\left(\left(K_{j} w_{x}\right) * K_{i}^{*}(z-x)\right) d z\right| \leq c \lambda 2^{-\eta s} s w(x),
$$

and

(2.2) for each fixed $x \in \sup B_{2, j-s}$,

$$
\left|\int \sum_{i \leq j-3} B_{2, i-s}(z)\left(\left(K_{j} w_{x}\right) * K_{i}^{*}(z-x)\right) d z\right| \leq c \lambda 2^{-\eta s} s w(x) .
$$

The former is easier to handle; it will be enough to prove

Lemma 2.3. Fix $s, j$, and $x \in \sup B_{1, j-s}$. Then for $i \leq j-3$, there exists $a$ decomposition $\left(K_{j} w_{x}\right) * K_{i}^{*}=k_{1, i}+k_{2, i}$, where

(i) $\left|k_{1, i}(z-x)\right| \leq c 2^{-2 j-s}|x|^{\alpha}$

(ii) $\left|\nabla_{z} k_{2, i}(z-x)\right| \leq c s 2^{-2 j-i}|x|^{\alpha}$.

Remark. This is a weighted version of Lemma 2 of $[\mathrm{H}]$; the estimate (2.1) follows from Lemma 2.3 in the same way that, in [H], estimate (2) follows from Lemma 2. We skip the details.

Before proving Lemma 2.3 we consider estimate (2.2). Fix $s, j$, and $x \in$ $\sup B_{2, j-s}$, and let $\chi\left(y^{\prime}\right)$ be the characteristic function of the set $\left\{y^{\prime} \in S^{1}: \| y^{\prime}-\right.$ $\left.x^{\prime} \| \leq 2^{-s / 4-(j-i) / 2}\right\}$. Write $\left(K_{j} w_{x}\right) * K_{i}^{*}=H_{1, i}+H_{2, i}$, where $H_{1, i}=\left(K_{j} \chi w_{x}\right) *$ $K_{i}^{*}$. We will prove (2.2) for $H_{1, i}$ and $H_{2, i}$ separately.

To handle the part of (2.2) corresponding to $H_{1, i}$, we observe that (with the normalization $\|\Omega\|_{\infty}=1$ )

$$
\begin{aligned}
\left|H_{1, i}(z-x)\right| & \leq \int\left|K_{j}(y) \chi\left(y^{\prime}\right)\right|\left|K_{i}^{*}(z-x-y)\right||y+x|^{\alpha} d y \\
& \leq 2^{-2 j-2 i} \int_{|y| \leq a|x|}\left|\chi\left(y^{\prime}\right)\right||y+x|^{\alpha} d y,
\end{aligned}
$$

which, by the same reasoning as in the proof of $(1.5)$ of $\S 1$, is bounded by

$$
c|x|^{\alpha} 2^{-2 i-\gamma(s / 4+[j-i] / 2)},
$$


for some positive constant $\gamma$. Now recall that by definition of $B_{2, i-s}$, we have $\sum_{n(i, s)}\left|Q_{n}\right| \leq c 2^{2 i}$, where the sum runs over those $Q_{n}$ contained in $\sup B_{2, i-s}$. Thus

$$
\begin{aligned}
& \left|\int \sum_{i \leq j-3} B_{2, i-s}(z) H_{1, i}(z-x) d z\right| \\
& \quad \leq \sum_{i \leq j-3} \sum_{n(i, s)}\left|\int b_{n}(z) H_{1, i}(z-x) d z\right| \\
& \quad \leq c|x|^{\alpha} \sum_{i \leq j-3} 2^{-2 i-\gamma(s / 4+[j-i] / 2)} \sum_{n(i, s)} \int\left|b_{n}(z)\right| d z .
\end{aligned}
$$

Since $\int\left|b_{n}\right| \leq c \lambda\left|Q_{n}\right|$, this last expression is bounded by

$$
c \lambda 2^{-\gamma s / 4}|x|^{\alpha} \sum_{i \leq j-3} 2^{-\gamma(j-i) / 2} .
$$

For $H_{1, i},(2.2)$ then follows.

To prove (2.2) for $H_{2, i}$, we will use

Lemma 2.4. Fix $s, j$, and $x \in \sup B_{2, j-s}$. Then for $i \leq j-3$, there exists $a$ decomposition $\left(K_{j}(1-\chi) w_{x}\right) * K_{i}^{*}=k_{1, i}+k_{2, i}$, where

(i) $\left|k_{1, i}(z-x)\right| \leq c 2^{-2 j-s-\alpha(s / 4+(j-i) / 2)}|x|^{\alpha}$

(ii) $\left|\nabla_{z} k_{2, i}(z-x)\right| \leq c s 2^{-2 j-i+(1-\alpha)(s / 4+(j-i) / 2)}|x|^{\alpha}$

We assume for now that Lemma 2.4 holds, and deduce (2.2). With $\sum_{n(i, s)}$ as above, and $z_{n}$ defined to be the center of $Q_{n}$, to prove (2.2) it is enough to estimate

$$
\sum_{i \leq j-3} \sum_{n(i, s)}\left(\int\left|b_{n}(z) k_{1, i}(z-x)\right| d z+\int\left|b_{n}(z)\left[k_{2, i}(z-x)-k_{2, i}\left(z_{n}-x\right)\right]\right| d z\right),
$$

where in the second integral we have used the fact that $b_{n}$ has mean value zero. The part of this expression involving $k_{1, i}$ is bounded by

$$
\begin{aligned}
& c|x|^{\alpha} \sum_{i \leq j-3} 2^{-2 j-s-\alpha(s / 4+[j-i] / 2)} \sum_{n(i, s)} \int\left|b_{n}(z)\right| d z \\
& \leq c \lambda 2^{-(1+\alpha / 4) s}|x|^{\alpha} \sum_{i \leq j-3} 2^{-(2+\alpha / 2)(j-i)},
\end{aligned}
$$

which implies the desired estimate since $\alpha>-2$. Similarly, the part involving $k_{2, i}$ is bounded by

$$
c s|x|^{\alpha} \sum_{i \leq j-3} 2^{-2 j-i+(1-\alpha)(s / 4+[j-i] / 2)} \sum_{n(i, s)} \int\left|b_{n}(z)\right|\left|z-z_{n}\right| d z,
$$

which, since $\left|z-z_{n}\right| \leq \operatorname{diam} Q_{n} \leq c 2^{i-s}$, is in turn less than or equal to

$$
c \lambda s 2^{-(1+(\alpha-1) / 4) s}|x|^{\alpha} \sum_{i \leq j-3} 2^{-(2+(\alpha-1) / 2)(j-i)} \text {. }
$$


Then (2.2) follows.

The proof of Theorem 1 has now been reduced to proving Lemma 2.3 and 2.4.

Proof of Lemma 2.3. As in the proofs of Lemmas 1.4 and 1.6, and with the same notation, we have the representation

$$
\left(K_{j} w_{x}\right) * K_{i}^{*}(z-x)=\iint \mu_{\theta, j, x} * \mu_{\omega, i}^{*}(z-x) d \theta d \omega
$$

where $\mu_{\theta, j, x} * \mu_{\omega, i}^{*}(z-x)$ is equal to

$2^{-j-i} \Omega(\theta) \Omega(\omega) \Delta^{-1} \psi\left(2^{-j} \omega^{\perp} \cdot(z-x) / \Delta\right) \psi\left(2^{-i} \theta^{\perp} \cdot(z-x) / \Delta\right) w_{x}\left(\left[\omega^{\perp} \cdot(z-x) / \Delta\right] \theta\right)$.

By the same reasoning as in the proof of Lemma 1.4, since $i<j$,

$$
\begin{aligned}
& \left|\nabla_{z} \mu_{\theta, j, x} * \mu_{\omega, i}^{*}(z-x)\right| \\
& \leq c \Delta^{-2}\left[2^{-j-2 i}\left|\left(\omega^{\perp} \cdot(z-x) / \Delta\right) \theta+x\right|^{\alpha}\right. \\
& \left.\quad+2^{-j-i}\left|\left(\omega^{\perp} \cdot(z-x) / \Delta\right) \theta+x\right|^{\alpha-1}\right] \\
& \leq c \Delta^{-2} 2^{-j-2 i}|x|^{\alpha} .
\end{aligned}
$$

Also,

$$
\left|\mu_{\theta, j, x} * \mu_{\omega, i}^{*}(z-x)\right| \leq 2^{-j-i} \Delta^{-1}|x|^{\alpha} .
$$

Set $\delta=2^{-s}$ and write $\left(K_{j} w_{x}\right) * K_{i}^{*}=k_{1, i}+k_{2, i}$, where

$$
k_{1, i}=\iint_{\|\theta-\omega\| \leq \delta} \mu_{\theta, j, x} * \mu_{\omega, i}^{*} d \theta d \omega .
$$

Since $i \leq j-3,\left(K_{j} w_{x}\right) * K_{i}^{*}(\cdot-x)$ is supported in $A_{j}(x)=\left\{z: 2^{j-3} \leq|z-x| \leq\right.$ $\left.2^{j+1}\right\}$. Thus, in (2.5), $|z-x| \approx 2^{j}$. Furthermore, by geometry,

$$
\Delta \approx\|\theta-\omega\| \text {. }
$$

Set $y^{\prime}=y /|y|$. By the support of $\psi$, the integrand in (2.5) is nonvanishing only if

$$
\Delta \approx 2^{-j}\left|\omega^{\perp} \cdot(z-x)\right| \approx\left|\omega^{\perp} \cdot(z-x)^{\prime}\right| \approx\left\|\omega-(z-x)^{\prime}\right\|
$$

and

$$
\Delta \approx 2^{-i}\left|\theta^{\perp} \cdot(z-x)\right| \approx 2^{j-i}\left|\theta^{\perp} \cdot(z-x)^{\prime}\right| \approx 2^{j-i}\left\|\theta-(z-x)^{\prime}\right\|
$$

Then

$$
\left|k_{1, i}(z, x)\right|
$$

$$
\leq c 2^{-j-i}|x|^{\alpha} \int \chi_{1}(\omega)\left\|\omega-(z-x)^{\prime}\right\|^{-1} \int \chi_{2}(\theta, \omega) d \theta d \omega,
$$

where $\chi_{1}(\omega)$ is the characteristic function of $\left\{\omega:\left\|\omega-(z-x)^{\prime}\right\| \leq c \delta\right\}$, and $\chi_{2}(\theta, \omega)$ is the characteristic function of $\left\{(\theta, \omega):\left\|\theta-(z-x)^{\prime}\right\| \approx 2^{i-j} \| \omega-\right.$ $\left.(z-x)^{\prime} \|\right\}$. Estimate (i) of Lemma 2.3 follows by straightforward computation. 
Estimate (ii) is now easy. With $\chi_{1}(\omega)$ and $\chi_{2}(\theta, \omega)$ as above,

$$
\begin{aligned}
& \left|\nabla_{z} k_{2, i}(z-x)\right| \leq \iint_{\|\theta-\omega\|>\delta}\left|\nabla_{z} \mu_{\theta, j, x} * \mu_{\omega, i}^{*}(z-x)\right| d \theta d \omega \\
& \quad \leq c 2^{-j-2 i}|x|^{\alpha} \int\left[1-\chi_{1}(\omega)\right]\left\|\omega-(z-x)^{\prime}\right\|^{-2} \int \chi_{2}(\theta, \omega) d \theta d \omega \\
& \quad \leq c 2^{-2 j-i}|x|^{\alpha} \log \delta^{-1} .
\end{aligned}
$$

This concludes the proof of Lemma 2.3.

Proof of Lemma 2.4. Again using the representation (2.5), we define

$$
\begin{aligned}
& k_{1, i}=\iint_{\|\theta-\omega\| \leq 2^{-s}} \mu_{\theta, j, x} * \mu_{\omega, i}^{*} d \theta d \omega, \\
& k_{2, i}=\iint_{\substack{\|\theta-\omega\|>x^{\prime}\|>\delta\\
\| \theta-x^{\prime} \|>\delta}}^{\substack{\| s, j, x \\
n^{-s}}} \mu_{\theta, i}^{*} d \theta d \omega,
\end{aligned}
$$

where $\delta=2^{-s / 4-(j-i) / 2}$.

Note that by definition of $\sup \psi$ and $\sup B_{2, j-s}$, we have

$$
|x| \approx 2^{j} \approx\left|\omega^{\perp} \cdot(z-x) / \Delta\right| .
$$

Thus for $\left\|\theta-x^{\prime}\right\|>2^{-s / 4-(j-i) / 2}$, we have

$$
\left|\left(\omega^{\perp} \cdot(z-x) / \Delta\right) \theta+x\right|>c 2^{j-s / 4-(j-i) / 2},
$$

and for $\alpha<0$,

$$
\left|\left(\omega^{\perp} \cdot(z-x) / \Delta\right) \theta+x\right|^{\alpha} \leq c 2^{\alpha(j-s / 4-(j-i) / 2)} \approx|x|^{\alpha} 2^{-\alpha(s / 4+(j-i) / 2)} .
$$

Recall also that

$$
\begin{aligned}
\left|\nabla_{z} \mu_{\theta, j, x} * \mu_{\omega, i}^{*}(z-x)\right| & \\
\leq c \Delta^{-2}\left[2^{-j-2 i} \mid\left(\omega^{\perp}\right.\right. & \cdot(z-x) / \Delta) \theta+\left.x\right|^{\alpha} \\
& \left.+2^{-j-i}\left|\left(\omega^{\perp} \cdot(z-x) / \Delta\right) \theta+x\right|^{\alpha-1}\right],
\end{aligned}
$$

which for $i<j,|x| \approx 2^{j}$, and $\left\|\theta-x^{\prime}\right\|>2^{-s / 4-(j-i) / 2}$, is in turn bounded by

$$
c 2^{-j-2 i+(1-\alpha)(s / 4+(j-i) / 2)} \Delta^{-2}|x|^{\alpha} \text {. }
$$

Similarly,

$$
\left|\mu_{\theta, j, x} * \mu_{\omega, i}^{*}(z-x)\right| \leq 2^{-j-i-\alpha(s / 4+(j-i) / 2)} \Delta^{-1}|x|^{\alpha} .
$$

The estimates for $\left|k_{1, i}\right|$ and $\left|\nabla k_{2, i}\right|$ are then obtained in the same way as the corresponding estimates in the proof of Lemma 2.3. This concludes the proof of Lemma 2.4, and hence also the proof of Theorem 1.

\section{ACKNOWLEDGMENTS}

The results described in this paper were part of the author's Ph.D. thesis, written at the University of Minnesota under the direction of Max Jodeit, Jr. I thank Professor Jodeit, and also the referee. 


\section{REFERENCES}

[CWZ] A. P. Calderon, M. Weiss, and A. Zygmund, On the existence of singular integrals, Proc. Sympos. Pure Math., Vol. 10, Amer. Math. Soc., Providence, R.I., 1967, pp. 56-73.

[C] M. Christ, Weak-type $(1,1)$ bounds for rough operators, Ann. of Math., 128, (1988), 19-41.

[CRdF] M. Christ and J. L. Rubio de Francia, Weak-type $(1,1)$ Bounds for Rough Operators II, preprint.

[H] S. Hofmann, Weak $(1,1)$ boundedness of singular integrals with nonsmooth kernel, Proc. Amer. Math. Soc. 103 (1988), 260-264.

[KW] D. S. Kurtz and R. Wheeden, A note on singular integrals with weights, Proc. Amer. Math. Soc. 81 (1981), 391-397.

[MW] B. Muckenhoupt and R. Wheeden, Weighted norm inequalities for singular and fractional integrals, Trans. Amer. Math. Soc. 161 (1971), 249-258.

[S1] E. M. Stein, Singular integrals and differentiability properties of functions, Princeton Univ. Press, Princeton, N.J., 1970.

[S2] _ Note on singular integrals, Proc. Amer. Math. Soc. 8 (1957), 250-254.

Department of Mathematics and Statistics, McMaster University, Hamilton, Ontario, Canada L8S 4K1 